\title{
Correlated Temporal and Spectral Variability
}

\author{
Jean H. Swank \\ NASA/GSFC Code 662, Greenbelt MD 20771, U.S.A.
}

\begin{abstract}
The variability of neutron star and black hole X-ray sources has several dimensions, because of the roles played by different important time-scales. The variations on time scales of hours, weeks, and months, ranging from $50 \%$ to orders of magnitude, arise out of changes in the flow in the disk. The most important driving forces for those changes are probably various possible instabilities in the disk, though there may be effects with other dominant causes. The changes in the rate of flow appear to be associated with changes in the flow's configuration, as the accreting material approaches the compact object, for there are generally correlated changes in both the Xray spectra and the character of the faster temporal variability. There has been a lot of progress in tracking these correlations, both for $\mathrm{Z}$ and Atoll neutron star low-mass X-ray binaries, and for black hole binaries. I will discuss these correlations and review briefly what they tell us about the physical states of the systems.
\end{abstract}

Keywords: accretion, accretion disks-black hole physics—-stars:neutron-X-rays:binaries PACS: 95.85.Nv, 97.60.Jd, 97.60.Lf, 97.80.Jp

\section{INTRODUCTION}

Accretion flow onto compact stars is complex. To understand the physical configurations we need to know many parameters: what are the emission regions, their sizes, the densities, the bulk velocities, the turbulent velocities, the temperatures? The emissions change a lot in flux and spectrum. Presumably there are dynamical changes occurring and these have the potential of telling us a lot about the parameters and the physics of the region. Mass, spin, and magnetic field must control what happens. To learn how, we have spectral and timing information. Possibly in the not too distant future we may have polarization information. Possibly in the very distant future interferometers may image the accretion region, but we hope to be able to use the indirect information to understand what is happening, however exciting images would be. In this paper I examine what we have been learning from having temporal and spectral characteristics at the same time and some special techniques that are used to learn more from the combined information. Our tools for examining the more complex situation are still relatively crude compared with our tools to analyze either alone.

The basic elements in an overview of binary compact objects are well known. External conditions deliver gas to the gravitational potential of the compact object. The companion star fills its Roche lobe or it has a stellar wind or the compact object is in an eccentric orbit and comes in close to the companion. Then there is both a large scale view of what happens in the case of either black holes or magnetic neutron stars and a view of the local physics that applies. Whether the local physics is the cause of what happens or it is driven by larger-scale forces is not generally easy to say.

The gas with angular momentum about the compact object goes into an accretion 
disk. Instabilities in the disk flow can make a surge of accretion and cause ripples. The radiation pressure can blow disk material into a corona. Magnetic fields can guide some charges normal to the disk, in a jet. Close to a black hole or a neutron star, General Relativity (GR) should modify the hydrodynamics and the dynamics.

Zooming into the local regions we encounter turbulence, oscillations, waves, and shocks. These may be the causes of the accretion, required for accretion to occur at all, but they show up in high frequency timing, such as shots, and quasi-periodic oscillations (QPO). Owen \& Blondin (1997) have simulated modes of an accretion disk. Balbus \& Hawley (1998) showed the disk is unstable, but the extended consequences of the instability continue to be worked out. So we have perturbations that are as loud as $30 \%$ to be diagnostic of the situation, but we still don't have a predictive understanding.

\section{SPECTRAL CORRELATIONS WITH LARGE SCALE CHANGES}

\section{Transients}

The most obvious markers of large scale changes are X-ray binary transients. Both black holes and neutron stars systems go from a luminosity comparable to that of an active flare star to a luminosity within an order of magnitude of the Eddington limit, or more, within a few days, and then subside back to quiescence over weeks to months or even years. Associated optical, infrared, and radio emission give us more information about the involvement of the accretion disk and the generation of outflows in jets.

In the cases in which Roche lobe overflow drives the transfer of matter from the companion star's surface to the disk or even when this is combined with capture of wind from the companion, an instability in the disk flow is believed to cause the sudden increase in the rate of flow through the disk and onto the compact object. This is successfully modeled in general, but the detailed differences in the light curves are still mysterious (e.g. Lasota 2001; Dubos, Hamreury, \& Lasota 2001).

The neutron star transients are of several different kinds. Those with strong magnetic fields are generally recently formed and most frequently have $\mathrm{Be}$ or O star companions. These stars have winds that are accreted, though the material still has angular momentum and may eventually form a disk. The neutron star may have been formed in an asymmetric explosion and have a very eccentric orbit, so that the wind it encounters varies as the distance from the star changes. Systems in which the magnetic field has decayed over time, with companions that are filling the Roche lobe of smaller orbits, have transient outbursts similar to those of the black hole transients. If the neutron star has enough of a magnetic field that the accretion is guided to the poles, the source is a millisecond pulsar.

GX 339-4 has had several outbursts. The 2002-2003 outburst led Homan \& Belloni (2005) (See also Belloni et al. 2005) to formulate a diagrammatic course of the outburst in a luminosity versus hardness diagram. The source starts out in a hard low state, rises to a high hard state, softens while still at high luminosity. Then it gradually ratchets down in luminosity while alternating between hardening and softening, until at a much reduced luminosity it again becomes as hard as in the initial state and retraces the path to the low and hard state again.

A 2005 outburst of GRO J1655-40 allowed a detailed multiwavelength following 
of the course of a black hole transient. Shaposhnikov et al. (2007) studied the rise with RXTE, INTEGRAL, the VLA, and ROTSE, supplemented by other optical observations. Radio and Optical brightening was detected within a day of the reported X-ray detection of the source for the first time since 1997. The X-ray flux rose by a factor of 20 with essentially the same hard spectrum until the spectrum started to soften with the appearance of an optically thick disk component in the spectrum. About 18 days after the first appearance the soft component and the hard component (approximately a power law) grew together for another week, until at the same time the disk flux jumped up, the hard component and the QPO power started down, and the radio flux disappeared below the level of the VLA sensitivity.

A recent publication by $\mathrm{Yu}$ et al.(2003) shows a similar type of behavior from the recurrent neutron star transient Aql X-1. In this case both soft and hard X-rays identify the start of the outburst, but there is an initial spike in the hard X-rays (HEXTE 15-30 $\mathrm{keV}$ ) during the first week, whereupon suddenly — within one day - the hard flux falls by a factor of 5 and the soft flux (tracked by the ASM) jumps up by a similar factor.

\section{Persistent X-Ray Binaries}

Persistent accreters actually look a lot like transients in having changes of state, The black hole binary LMC X-1 has a supergiant companion driving a wind that is accreted, but it is close to the Roche lobe also and hard and soft states are observed in the X-ray flux, which are perhaps the result of changes of physical state similar to those of the transients. Boyd et al. (2000) found that the variations in ASM count rate correspond to dramatic flipping between a hard power law and a soft thermal emission.

The persistent low-mass binary neutron star sources are well known for their $Z$ and Atoll shaped excursions in a hard versus soft color diagram and in the hard color versus luminosity diagram. (See review by van der Klis (1989).) Thus the $Z$ sources transit between Horizontal, Normal, and Flaring branches and the Atoll sources move around an intermittent circular pattern between an Island state and the "Lower Banana" and "Upper Banana" sections of the diagram. Wijnands (2001) summarized how these look in RXTE observations.

In the transients, at least, there is real change in the amount of accretion flow during the outburst. So it is natural to conclude that the spectral variations depend on the mass accretion rate. Plausibly there are several accretion rates that are relevant, e.g. an average rate, the rate of transfer from the companion to a disk, the rate from a disk to the compact object, the rates of flow through the disk at various radii, the rate of flow from a disk to a coronal distribution, the rate of outflow, the rate of fall of coronal material. In the development of changes there can be hysteresis in the action of different components (e.g. Smith et al. (2001)), which could come from different timescales associated with them.

The spectra depend on the magnetic field, in the sense that different classes look different, though they also have different accretion rates, so that these effects may be entangled.

There are differences between black hole and neutron star sources, although there 
are many similarities. The two compact objects are expected to be different in terms of the existence of a hard surface in which falling material is thermalized. The range of luminosity of massive black holes is of course larger. Black holes have perhaps been spun to higher angular momentum states. These things can influence the size of the emission region, the optical depths of material and these influence the spectra, time delays, and dynamics.

\section{TEMPORAL CORRELATIONS WITH STATE CHANGES}

Corresponding to the spectral characteristics of states are temporal characteristics. Again there are similarities between the black hole and the neutron star behaviors.

In the outburst of GRO J1655-40 referred to above, we found a QPO at low frequency $(0.02 \mathrm{~Hz})$ present at the lowest luminosity. This frequency rose proportionally to the fluxes to a maximum of $16 \mathrm{~Hz}$ and disappeared at the transition to a high soft state, a few days after the radio became undetectable. The presence of quite strong low frequency QPO in the hard states and the lack of it in the soft or thermally dominant states is well known. The disk is not turbulent, or has red noise, while the corona has strong oscillations. In detail the QPO may be several phenomena. As discussed by Remillard et al. (2002), distinctions are made depending on the structure of the feature in the power spectra, the relative strength of the first harmonic and an apparent sub-harmonic of the strongest peak, lags between the hard and soft photons, and their coherence.

The transient mentioned above, Aql X-1, is analagous to black hole transients in its temporal behavior as well as its spectral behavior. Yu et al. show that the soft state has a power spectrum dominated by red noise, while the hard initial spike has band-limited white noise with a QPO. The frequency is $10 \mathrm{~Hz}$ instead of $<1 \mathrm{~Hz}$, in accordance at least qualitatively with scaling inversely as the mass of the compact object.

Comparison of the highest frequencies of the black hole sources and the neutron star sources is a matter of some controversy, as to whether the phenomena are the different, the same, or partly the same. There are relatively few cases of high frequencies in the power spectra of black hole sources because it only appears in rare states, while most bright low-mass binary neutron star sources have exhibited $\mathrm{kHz}$ oscillations at a range of luminosities and spectral states. The signal strength of these oscillations is an order of magnitude stronger for neutron star accretion than black holes. Even so it remains a challenge to understand the behavior. But several comprehensive studies are establishing characteristics which are restricting models and are meaningful tests of models.

The neutron star sources have shown different relations of flux to frequency at different times. We have expected the physical response to be responding to the accretion rate rather than the X-ray flux. The characteristics of several phenomena support the idea that there is not a one to one correspondence between flux observed and the accretion rate. Color-color diagrams are independent of flux and a variable can be assigned to the position in the diagram - a hidden variable, so to speak, called $S_{a}$, first used by Hasinger et al. (1990). As Mendez (2000) showed, QPO frequencies as functions of that variable are approximately independent of source and of "track". This procedure is unsatisfactory in having an anonymous fundamental parameter. Also, the correspondence is imperfect; there must be other things that matter. But one of the other characteristics independent 
of track, but a function of frequency, is the fractional root mean square (rms) variability (Mendez et al. 2001). This ruled out a model in terms of two different accretion flows, one participating in the oscillations and one not, in that while for a given frequency there were observations with two different fluxes, they had the same fractional rms variability.

Since the spectrum determines $S_{a}$ and the frequency depends on $S_{a}$, the frequency itself can taken as a surrogate for whatever physical parameter is determining the system. As the fractional rms variability depends on the frequency, so too does the quality of the $\mathrm{QPO}$, defined as $v / \delta v$. Barret, Olive, and Miller $(2006,2007)$ have shown that for 6 sources, the lower and upper $\mathrm{kHz} \mathrm{QPO}$ have similar behavior with frequency, regardless of luminosity within that source. The lower QPO can have Q of 100-200. The upper QPO hardly reaches 50 . In this representation it is convincing that for a given source there is a maximum lower $\mathrm{kHz} \mathrm{QPO}$ frequency - where $\mathrm{Q}$ vanishes. The demarcation for the high frequency QPO is more difficult to detect as Q becomes smaller. But to the extent that the upper and lower $\mathrm{kHz}$ frequencies have relatively specific separation, it too has a maximum. Thus there appears to be a cutoff which is unique to a source. This would be consistent with its being associated with an innermost stable circular orbit (ISCO). The authors argue that the unique radius should not be that of the neutron star because modern theory results in neutron stars with radii inside the minimum stable orbits or a magnetosphere radius because it should depend on the luminosity.

Thus the connection between the spectrum and the frequency in this case leads to fundamental inferences for the configuration and the physics. It would be consistent with the highest frequencies of the two QPO being associated with an orbit acting like the ISCO does in theory. If this association can be made, the frequency identifies the orbit and the mass of the neutron star within it.

An alternative to multiple modes of accretion could be that the frequency and the spectrum depend on the radius of the orbit, while there can be different rates of inflow through the ring at that radius. Fig. 1 shows a hypothetical dependence of the freqency and the hardness on a parameter $\mathrm{r}_{i}$, the supposed underlying physical quantity, which might not literally be the radius of the innermost stable circular orbit, but related to it. The X-ray luminosity would then indeed represent a mass flow to the neutron star. The "hard" color is not monotonically related to $r_{i}$, because it starts high in the Island state, is low when the source in in the Lower Banana state and rises again as it approaches the Upper Banana. However in terms of hard and soft colors the source is at unique points in a color-color diagram and the spectra, while they can be described in terms of several mathematical models, are different for observations falling in different spots of the Atoll diagram. Mass that is not expelled in an outflow should fall on the neutron star and release gravitational energy for emitting X-rays.

The spectrum could be determined by the energy release of the plasma falling from $\mathrm{r}_{i}$ and other gas that scatters it. The mechanism of the spectral formation would have to allow the spectrum to not be strongly influenced by the amount of the gas participating. It has been assumed in some models of low-mass X-ray binaries that the spectrum is formed by up-scattering in hot gas, a mechanism that seems to describe the formation of spectra in accreting binary black holes. It is not clear that the same spectrum could be produced for different rates of flow, because this might influence quantities like the optical depths and cooling rates that in turn might influence the spectrum.

The interpretation by Barret, Olive, and Miller of the decline of $Q$ and the rms 


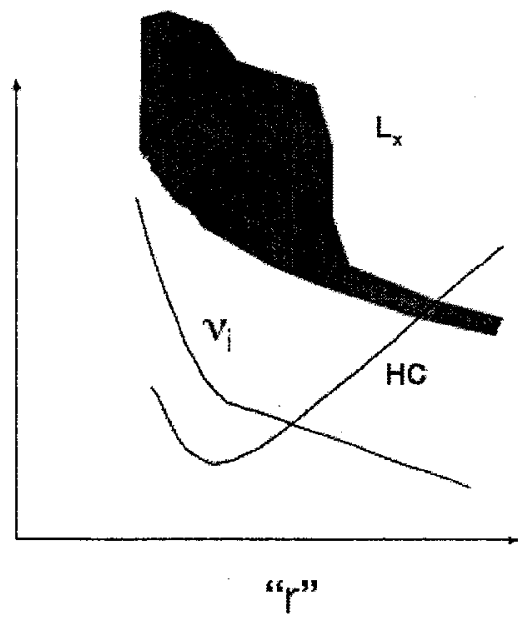

FIGURE 1. Dependences of observables on a Physical Parameter. $\mathrm{L}_{x}, v$, and the hard color of the spectrum depend on $S_{a}$ which can be defined in terms of a length along the color-color diagram. This could be related to a physical parameter " $r$ ", perhaps related to the inner radius, $r_{i}$, of the accretion disk. The figure is a sketch of possible relations of the X-ray luminosity, the frequency, and a spectral hardness on $r_{i}$

variability of an individual source in terms of the influence of the ISCO has been cast in a different light by Mendez (2006). It is also true that as the average luminosity of sources increases, from Atoll sources with $\mathrm{L} / \mathrm{L}_{E d d} \leq 0.2$ to $\mathrm{Z}$ sources with near Eddington luminosities for a neutron star mass, the maximum $Q$ and the rms also decline. Mendez raises the possibility that the driving influence is the hardness of the spectrum and that the individual and the ensemble behavior has the same cause, which would not be the influence of the ISCO .

In the scenario I have outlined above the effects would have different causes and the hardness would be a function of the situation of the accretion, the potential drop of the infalling material and the density of the plasma in the environment. This discussion has not addressed why the ISCO could be reached at different luminosities for different sources. The magnetic field is so far glaringly neglected. The binary periods range from less than an hour to days so that the accretion disks are of different sizes. Both these effects should influence the environment. I would expect the decline in individual sources to be different quantitatively than the decline of the maxima in the ensemble if the effects are different.

Recently Barret, Olive, and Miller (2007) have emphasized that in the case of $4 \mathrm{U}$ 1636-536, all the action of $Q$ versus frequency takes place at approximately constant hardness. In other sources, QPO are present for a range of hardness, but in 4U 1636536 , at least, clear changes of spectrum do not occur at the cutoff of the QPO. The dependences of $\mathrm{Q}$ and rms on frequency for individual sources appear clear for at least 5 sources and more are under study. The mechanisms are not yet clear and a picture encompassing the variety of sources is not yet clear. 


\section{SPECTRAL CORRELATIONS WITH TEMPORAL VARIABILITY}

The power spectra of the X-ray flux on time scales from minutes to milliseconds reflect what is happening relatively close to the compact objects. The fractional amplitudes of most of the QPO increase as a function of the photon energy. This is true for black hole sources where the model is a disk and a coronal component. Thus the photons emerging from the corona are modulated, although in some cases the amplitude is well correlated with the disk luminosity. It is also true of the LMXB neutron stars where the contributions from both the neutron star surface and the inner disk are at issue.

Gilfanov, Revnivtsev \& Molkov (2003) [10] attempted to find the energy spectrum of the oscillating photons. They made power spectra for small energy bins $(1 \mathrm{keV})$ and took the amplitude of defined frequency ranges of each of those. From that they constructed for a given frequency range the energy spectrum. This led them to conclude that the energy spectrum of the high frequencies was a Compton up-scattered optically thick component from the neutron star surface, which they considered to be a boundary layer of the accreting material with the neutron star. On the other hand the non-varying component was a soft spectrum which they took to be an optically thick spectrum from the inner disk, of lower temperature. This work corresponded to similar studies of Cyg X-1 (Churazov, Gilfanov \& Resnivtsev 2001). [7] More recently, the technique was applied to study the contribution of the boundary layer in different sections of the $\mathrm{Z}$ diagram (Gilfanov \& Resnivtsev 2005). [8] This approach has been most applicable to the luminous sources with spectra suggesting independent components.

For neutron stars the fast varying flux has been interpreted as coming from the "boundary layer". However, it can still be possible that the variations are caused by dynamics in the disk. For examples, something in the disk could occult the boundary layer emission or variations in the disk could modulate the rate of matter impinging on the boundary layer. Whether the disk could cause the variations without the disk flux itself varying is not clear.

A different approach to the question of the relation between the variations and the spectra is to find the time delays between energy bands. Comptonization of soft photons by high temperatures or by energetic electrons should make the hard photons lag behind the soft flux. For neutron star QPO the opposite happens; the hard photons lead and the soft lag (Kaaret 1999) [13] In the case of black hole QPO, both cases have been found, for different types of QPO. While the amounts of lag can be interpreted in terms of a size of the emission region - consistent with ISCOs - there is little understanding of how this is accomplished consistent with the spectral results.

\section{CONCLUSIONS}

Long term variations come from large scale phenomena which can affect the whole disk. They cause configuration changes, for example between geometrically thin disk and geometrically thick corona, and the consequent spectral changes

Short time-scale, or high frequency variations, depend on the configurations, as do the spectra, so they are correlated to the spectra that are averaged over the short time-scales. These frequencies are dynamical, with or without GR, so they have the potential to be 
indicative of GR, if it is strong, and some modes of oscillation might only exist in GR.

The short time-scale variations have their own spectra. That is, the oscillating flux has a spectrum that seems to be different from the average spectrum in most cases that have been studied. This is perhaps the ultimate microscope that temporal and spectral correlations can apply, but it is not easy to draw unique conclusions. Its power has probably not yet been fully utilized.

In summary, correlated temporal and spectral variability have added a lot to our understanding of accretion onto binary compact objects and we are continuing to use them to further explore these systems.

\section{ACKNOWLEDGMENTS}

The astrophysics community has been very supportive of RXTE as a mission so far uniquely capable of studying the hectohertz to kilohertz variability of compact X-ray sources over a broad energy range and NASA's support of RXTE's continued operations is allowing deep investigations of the many phenomena discussed in this paper.

\section{REFERENCES}

1. Balbus, S. A., \& Hawley, J. F. 1998, Reviews of Modern Physics, 70, 1

2. Barret, D., Olive, J.-F., \& Miller, M. C. 2005, MNRAS, 361, 855

3. Barret, D., Olive, J.-F., \& Miller, M. C. 2006, MNRAS, 370, 1140

4. Barret, D., Olive, J.-F., \& Miller, M. C. 2007, MNRAS, 376, 1139

5. Belloni, T., Homan, J., Casella, P., van der Klis, M., Nespoli, E/., Lewin, W. H. G.,Miller, J. M., \& Mendez, M. 2005, Astronomy \& Astrophysics, 440, 207

6. Boyd, P. T., Smale, A. P., Homan, J., Jonker, P. G., van der Klis, M., \& Kuulkers, E. 2000, Astrophysical Journal (Letters), 542, L127

7. Churazov, E., Gilfanov, M., \& Revnivtsev, M. 2001, MNRAS, 321, 759

8. Gilfanov, M., \& Revnivtsev, M. 2005, Astronomische Nachrichten, 326, 812

9. Dubus, G., Hameury, J.-M., \& Lasota, J.-P. 2001, Astronomy \& Astrophycics, 373, 251

10. Gilfanov, M., Revnivtsev, M., \& Molkov, S. 2003, Astronomy \& Astrophysics, 410, 217

11. Hasinger, G., van der Klis, M., Ebisawa, K., Dotani, T., \& Mitsuda, K. 1990, Astronomy \& Astrophysics, 235,131

12. Homan, J., \& Belloni, T. 2005, Astrophysics and Space Science, 300, 107

13. Kaaret, P., Piraino, S., Ford, E. C., \& Santangelo, A. 1999, Astrophysical Journal (Letters), 514, L31

14. Lasota, J.-P. 2001, New Astronomy Review, 45, 449

15. Méndez, M. 2000, Nuclear Physics B Proceedings Supplements, 80, 1516

16. Méndez, M., van der Klis, M., \& Ford, E. C. 2001, Astrophysical Journal, 561, 1016

17. Méndez, M. 2006, MNRAS, 371, 1925

18. Owen, M. P., \& Blondin, J. M. 1997, IAU Colloq. 163: Accretion Phenomena and Related Outflows, 121,779

19. Remillard, R. A., Sobczak, G. J., Muno, M. P., \& McClintock, J. E. 2002, Astrophysical Journal, 564,962

20. Shaposhnikov, N. , Swank, J. , Shrader, C. R., Rupen, M., Beckmann, V., Markwardt, C. B., \& Smith, D. A. 2007, Astrophysical Journal, 655, 434

21. Smith, D. M., Heindl, W. A., \& Swank, J. H. 2002, Astrophysical Journal, 569, 362

22. van der Klis, M. 1989, Annual Reviews of Astronomy and Astrophysics, 27, 517

23. Wijnands, R. 2001, Advances in Space Research, 28, 469

24. Yu, W., Klein-Wolt, M., Fender, R., \& van der Klis, M. 2003, Astrophysical Journal (Letters), 589, L33 